\title{
FIRST RESULTS ON THE EXCITED STATES IN ${ }^{77} \mathrm{Cu}^{*}$
}

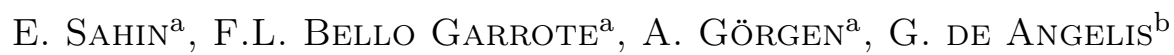

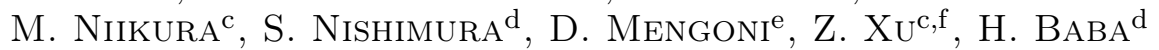
F. Browne ${ }^{\mathrm{d}, \mathrm{g}}$, P. Doornenbal ${ }^{\mathrm{d}}$, S. Franchoo $^{\mathrm{h}}$, G. Gulllaume $^{\mathrm{d}, \mathrm{i}}$

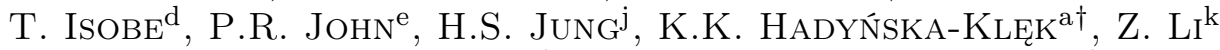

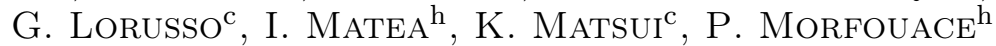
D.R. NAPOli ${ }^{\mathrm{b}}$, H. Nishibata ${ }^{\mathrm{l}}$, A. Odahara ${ }^{l}$, H. SAKURAI $^{\mathrm{c}, \mathrm{d}}$ P.-A. Söderström ${ }^{\mathrm{d}}$, D. SOhler ${ }^{\mathrm{m}}$, I. Stefan ${ }^{\mathrm{h}}$, T. SumikAma $^{\mathrm{n}}$ D. SUZUKI ${ }^{\mathrm{h}}$, R. TANIUCHI ${ }^{\mathrm{c}}$, J. TAPROGGE $^{\mathrm{d}, \mathrm{o}}, \mathrm{Z}$. VAJTA ${ }^{\mathrm{d}, \mathrm{m}}$

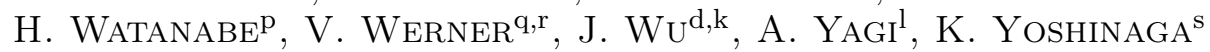

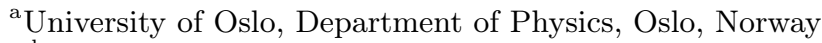

${ }^{\mathrm{b}}$ INFN Laboratori Nazionale di Leganro, Padova, Italy

${ }^{\mathrm{c}}$ Dept. of Physics, University of Tokyo, Hongo 7-3-1, Bunkyo-ku, 113-0033 Tokyo, Japan

${ }^{\mathrm{d}}$ RIKEN Nishina Center, 2-1 Hirosawa, Wako, Saitama 351-0198, Japan

${ }^{\text {e} I N F N ~ S e z . ~ d i ~ P a d o v a ~ a n d ~ D i p a r t i m e n t o ~ d i ~ F i s i c a, ~ U n i v . ~ d i ~ P a d o v a, ~ P a d o v a, ~ I t a l y ~}$

${ }^{\mathrm{f}}$ University of Hong Kong, Hong Kong

${ }^{\mathrm{g}}$ School of Computing, Engineering and Mathematics, Univ. of Brighton Brighton, BN2 4GJ, UK

${ }^{\mathrm{h}}$ Institut de Physique Nucleaire (IPN), IN2P3-CNRS, Univ. Paris-Sud 11 91406 Orsay Cedex, France

${ }^{\mathrm{i}}$ LPSC, Universite Joseph Fourier, CNRS/IN2P3

Institut National Polytechnique de Grenoble, France

${ }^{j}$ Dept. of Physics, University of Notre Dame, Notre Dame, Indiana 46556, USA

${ }^{\mathrm{k}}$ Dept. of Physics, Peking University, Beijing 100871, China

${ }^{1}$ Dept. of Physics, Osaka Univ., 1-1 Machikaneyama, Toyonaka, Osaka 560-0043, Japan

${ }^{\mathrm{m}}$ Institute for Nuclear Research of the Hungarian Academy of Sciences Debrecen 4001, Hungary

${ }^{\mathrm{n}}$ Dept. of Physics, Tohoku University, 6-3 Aramaki-Aoba, Aoba, Sendai 980-8578, Japan

${ }^{\circ}$ Instituto de Estructura de la Materia, CSIC, 28006 Madrid, Spain

${ }^{\mathrm{P}}$ International Research Center for Nuclei and Particles in the Cosmos Beihang University, Beijing, China

${ }^{\mathrm{q}}$ Wright Nuclear Structure Laboratory, Yale University New Haven, CT 06520-8120, USA

${ }^{\mathrm{r}}$ Institut für Kernphysik, Technische Universität Darmstadt, 64289 Darmstadt, Germany

${ }^{\mathrm{s}}$ Dept. of Physics, Tokyo Univ. of Science, 2641 Yamazaki, Noda, Chiba 278-8510, Japan

(Received December 18, 2015)

The level structure of the ${ }^{77} \mathrm{Cu}$ nucleus has been studied by means of $\beta$-delayed $\gamma$-ray spectroscopy at RIKEN Nishina Center. Secondary beam

* Presented at the XXXIV Mazurian Lakes Conference on Physics, Piaski, Poland, September 6-13, 2015.

† Current affiliation: INFN Laboratori Nazionale di Leganro, Padova, Italy. 
particles in the region near ${ }^{78} \mathrm{Ni}$ were produced via in-flight fission of ${ }^{238} \mathrm{U}$ on a thick ${ }^{9}$ Be target at a primary beam energy of $345 \mathrm{MeV} /$ nucleon. After identification in atomic number $(Z)$ and mass-to-charge ratio $(A / Q)$ in the BigRIPS fragment separator, the fission products were delivered to the final focal plane through the ZeroDegree Spectrometer. The WAS3ABI silicon stack array was used for the implantation of the ions, while de-excitation $\gamma$ rays were detected with the EURICA $\gamma$-ray spectrometer at the focal plane. Beta-correlated particle identification of ${ }^{77} \mathrm{Ni}$ allowed to obtain the $\gamma$-ray spectrum of the daughter ${ }^{77} \mathrm{Cu}$ in a selective way. Experimental details and first results on the excited states in ${ }^{77} \mathrm{Cu}$ nucleus will be presented in this contribution.

DOI:10.5506/APhysPolB.47.889

\section{Introduction}

The search for possible modifications of the shell structure in the ${ }^{78} \mathrm{Ni}$ region has been subject of a wide range of experimental studies over the last decade. The tensor component of the nuclear force is expected to shift the single-particle energies depending on the filling of specific orbitals. Such energy shifts can reduce shell gaps and lead to enhanced collectivity. Shell model calculations that include the tensor interaction predict an inversion of the $1 f_{5 / 2}$ and $2 p_{3 / 2}$ proton orbitals for the $\mathrm{Ni}$ isotopes between $N=40$ and $N=50$ as more neutrons fill the $1 g_{9 / 2}$ orbital [1, 2]. Here, the tensor force acts attractive between two orbits with anti-parallel spin configuration and repulsive for those with parallel spin directions. In addition, the $Z=28$ shell gap was predicted to be smaller towards $N=50$ due to the same mechanism as a result of the attraction between protons in $1 f_{5 / 2}$ and neutrons in $1 g_{9 / 2}$, and repulsion between protons in $1 f_{7 / 2}$ and neutrons in $1 g_{9 / 2}$. The heavier $\mathrm{Ni}$ isotopes $(N>46)$, however, are difficult to access via existing spectroscopic techniques and the investigation of the shell evolution is not completed over the whole $\mathrm{Ni}$ chain. In this context, neutron-rich $\mathrm{Cu}$ nuclei with one proton outside of the $Z=28$ shell and neutron numbers between $N=40$ and 50 constitute a good probe to see changes in the shell structure close to ${ }^{78} \mathrm{Ni}$. For instance, the mentioned inversion of the $\pi 1 f_{5 / 2}$ and $\pi 2 p_{3 / 2}$ orbits was experimentally proven by observing the ground-state spin-parity of ${ }^{75} \mathrm{Cu}$ to be $5 / 2^{-}$instead of $3 / 2^{-}$as in the lighter $\mathrm{Cu}$ nuclei [3]. Also a value of $4.7(3) \mathrm{MeV}$ for the $N=50$ shell gap could be determined in a recent high-spin work on the neutron rich ${ }^{83} \mathrm{As},{ }^{82} \mathrm{Ge}$, and ${ }^{81} \mathrm{Ga}$ nuclei [4].

Previous $\beta$-decay, Coulomb excitation, and multi-nucleon transfer reaction studies [5-9] characterized excited states in the lighter $\mathrm{Cu}$ isotopes (up to $\left.{ }^{73} \mathrm{Cu}_{44}\right)$ as single-particle and core-coupling excitations. The low-lying $5 / 2^{-}$and $7 / 2^{-}$states were identified to be the states of predominant singleparticle character from the $1 f_{5 / 2}$ and $1 f_{7 / 2}$ orbitals, respectively, while the 
lowest-lying $1 / 2^{-}$state was found to be collective. A second type of excitation was observed for the remaining low-lying $7 / 2^{-}$states, explained as a coupling of one proton in $1 f_{5 / 2}$ or $2 p_{3 / 2}$ to the $2^{+}$state of the even-even "core" nucleus, ${ }^{(68-72)} \mathrm{Ni}[7,9]$.

Experimental data on excited states are very limited for the heavier $\mathrm{Cu}$ isotopes beyond ${ }^{73} \mathrm{Cu}$. In addition to the experimental observation of the $5 / 2^{-}$ground-state spin-parity [3], two low-lying microsecond isomeric states were observed in ${ }^{75} \mathrm{Cu}[10,11]$. For ${ }^{77} \mathrm{Cu}$, a $5 / 2^{-}$ground-state spin and parity assignment was proposed in the $\beta$-decay work of Patronis et al. [12] and soon after of Ilyushkin et al. [13]. In the present paper, we report on the identification of low-lying excited states in ${ }^{77} \mathrm{Cu}_{48}$, which allows us to trace the evolution of the single-particle and collective properties towards the $Z=28$ and $N=50$ shell closures.

\section{Experimental details}

The recent experimental study of ${ }^{77} \mathrm{Cu}$ was carried out at RI Beam Factory (RIBF) of the RIKEN Nishina Center [14]. Data were taken in two different experiments exploiting the same primary beam and target combinations. Exotic secondary beam particles in the vicinity of ${ }^{78} \mathrm{Ni}$ were produced via fission reaction of the ${ }^{238} \mathrm{U}$ primary beam on a thick ${ }^{9} \mathrm{Be}$ target. The uranium beam was accelerated to an energy of $345 \mathrm{MeV} /$ nucleon with an average beam intensity of $10 \mathrm{pnA}$ in both experiments. The beryllium production targets had a thickness of 3 and $2 \mathrm{~mm}$ in the first and second experiment, respectively. After the production and selection, the secondary beam particles were identified in atomic number $(Z)$ and mass-to-charge ratio $(A / Q)$ using the $\mathrm{TOF}-B \rho-\Delta E$ technique [15] at the second stage of the BigRIPS fragment separator $[16,17]$. Ions were, then, delivered to the experimental setup through the ZeroDegree Spectrometer (ZDS) [17]. The experimental setup consisted of the EURICA Germanium array [18, 19] surrounding the stack of silicon detectors, called WAS3ABI [18]. WAS3ABI was used for ion implantation as well as for the detection of $\beta$-decay and internal conversion electrons. It was made of 8 Double-Sided Silicon Strip Detectors (DSSSD) with $1 \mathrm{~mm}$ thickness in each layer, segmented into sixty and forty strips in $X$ and $Y$ directions, respectively. The $\gamma$ rays emitted after $\beta$ decay of the implanted ions were detected by EURICA (EUroball RIken Cluster Array). It comprises of 84 High-Purity Germanium (HPGe) detectors organized in 12 clusters ( 7 crystals each) providing a $\gamma$-ray efficiency of $13 \%$ at $1 \mathrm{MeV}$ in the current configuration. An aluminum degrader was used before the ions arrive in the focal plane in order to allow the implantation to occur in one of the middle layers of the WAS3ABI silicon stack. Figure 1 shows a schematic view and a photo of the setup. 

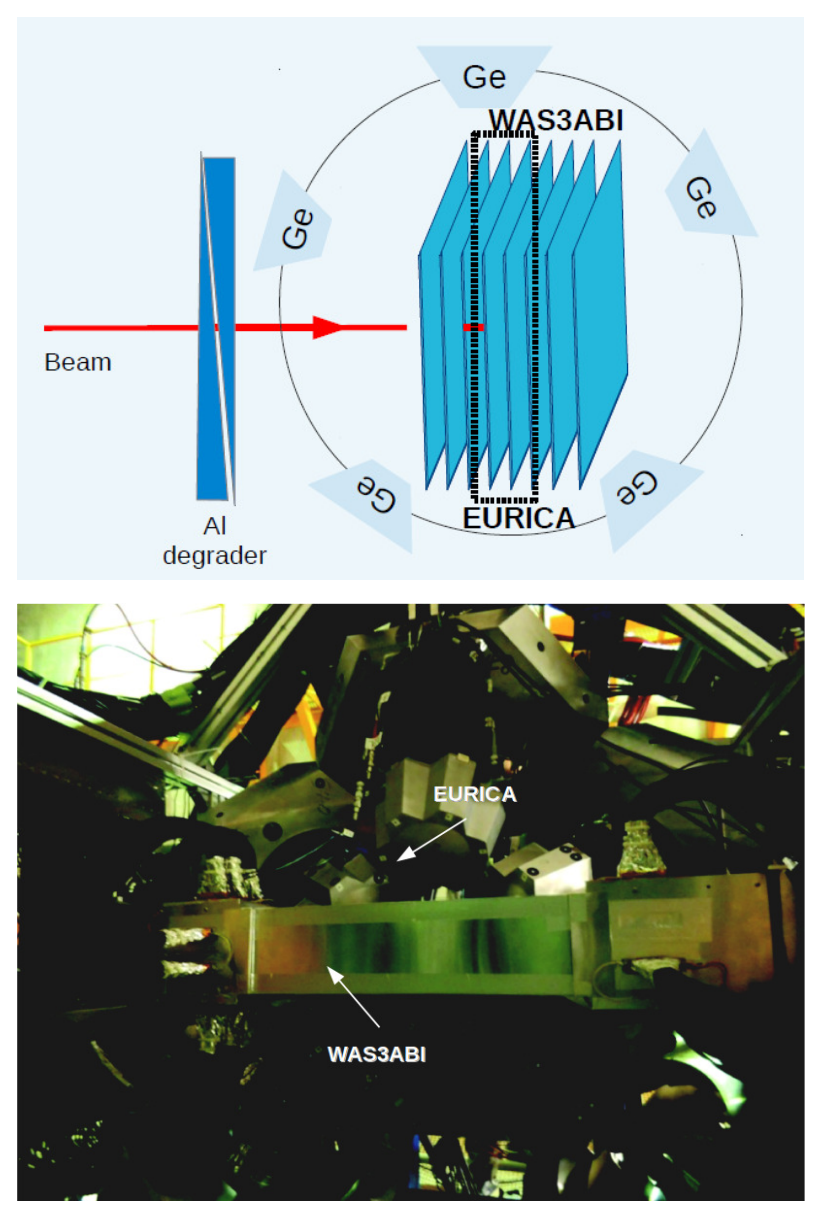

Fig. 1. (Upper panel) A schematic view of the setup. (Bottom panel) A photo of the setup taken during the experiment.

\section{Experimental results}

Excited states in ${ }^{77} \mathrm{Cu}$ were populated via $\beta$ decay of ${ }^{77} \mathrm{Ni}$. Correlations of the implanted ${ }^{77} \mathrm{Ni}$ ions with their subsequent $\beta$ decays to the daughter ${ }^{77} \mathrm{Cu}$ nuclei were constructed on an event-by-event basis. Up to two neighbouring pixels on the same silicon layer were included as a source of electron interaction points in order to increase the correlation efficiency. Figure 2 shows the particle identification (PID) of the correlated events in $Z$ as a function of $A / Q$. 


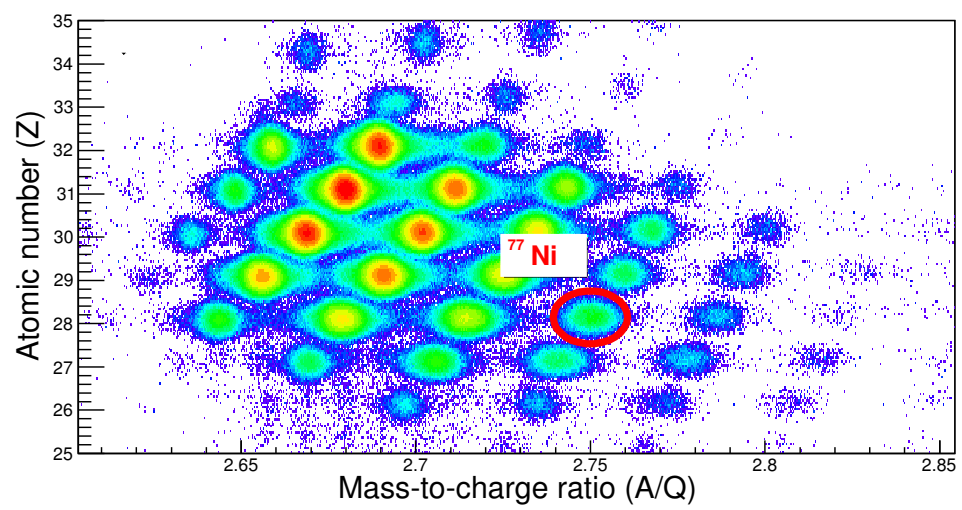

Fig. 2. Particle identification in $Z$ as a function of $A / Q$ after selecting only $\beta$-decay events.

A careful selection of the ${ }^{77} \mathrm{Ni}$ nuclei in the PID matrix is used to produce a clean $\gamma$-ray spectrum for transitions in the daughter nucleus ${ }^{77} \mathrm{Cu}$. Figure 3 shows such a spectrum in which the $\gamma$ rays of ${ }^{77} \mathrm{Cu}$ are clearly observed. These $\gamma$ rays are indicated with their energy values in the spectrum. Few lines arising from either the sequential decays of the daughter nucleus or the $\beta$-delayed neutron emission process are labelled with their element number, while those which we did not succeed to identify are indicated with a star.

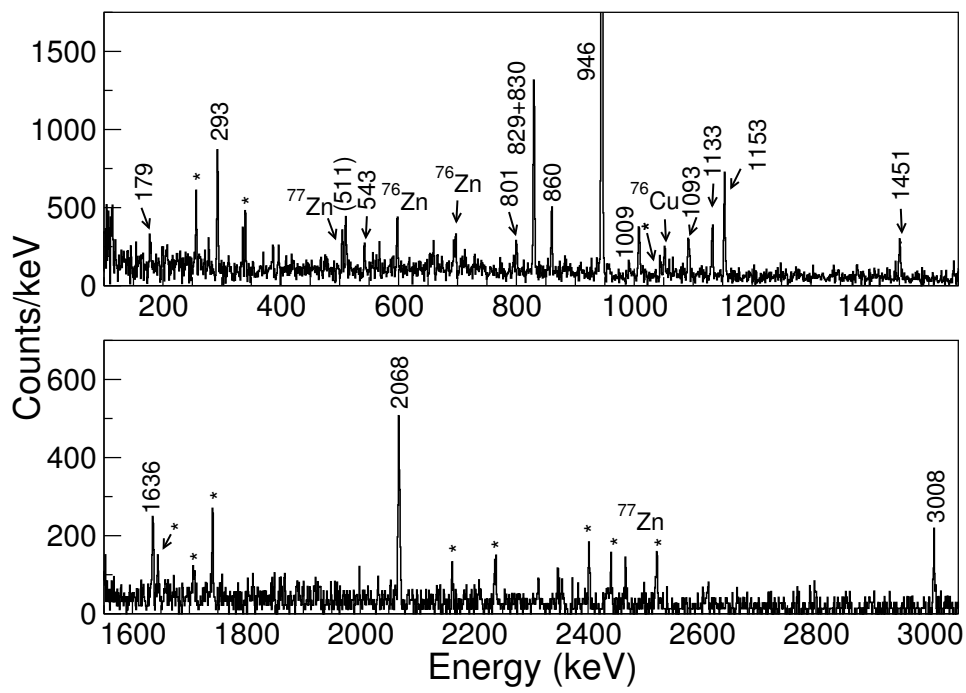

Fig. 3. $\gamma$-ray spectrum of ${ }^{77} \mathrm{Cu} . \quad \gamma$ rays assigned for ${ }^{77} \mathrm{Cu}$ are labelled with their values. The contamination lines from the successive decays are labelled with the name of the emitting nuclei, while lines which cannot be assigned to any nuclei in the decay chains are labelled with a star. 
The statistics collected during the two experiments was sufficient to analyse $\gamma-\gamma$ coincidences. A careful analysis of the coincidence relationships between the observed transitions will be used to construct the level scheme of ${ }^{77} \mathrm{Cu}$. An example of a $\gamma$-ray energy spectrum gated on the $946 \mathrm{keV}$ line is given in Fig. 4.

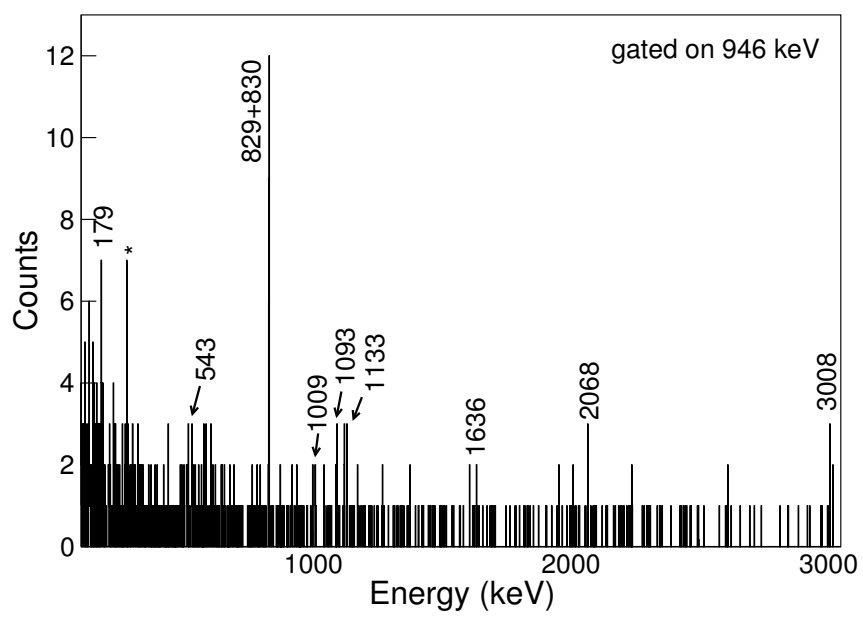

Fig. 4. $946 \mathrm{keV}$ gated $\gamma$-ray energy spectrum. Contamination lines are labelled with a star.

\section{Summary and conclusions}

In summary, $\gamma$-ray spectroscopy of neutron-rich ${ }^{77} \mathrm{Cu}$ was performed through the $\beta$-decay reaction of ${ }^{77} \mathrm{Ni}$ at RIKEN Nishina Center. The secondary beam of ${ }^{77} \mathrm{Ni}$ was identified in atomic number and $A / Q$ ratio by the BigRIPS fragment separator and implanted in the WAS3ABI silicon array. After the correlation between implanted ${ }^{77} \mathrm{Ni}$ ions and their subsequent $\beta$-decay electrons, $\gamma$ rays emitted from ${ }^{77} \mathrm{Cu}$ were detected by $12 \mathrm{HPGe}$ clusters of the EURICA array. $\gamma$-ray transitions of ${ }^{77} \mathrm{Cu}$ were obtained by selecting the $\beta$-correlated Ni events on even-by-event basis. Sufficient $\gamma-\gamma$ coincidence data made it possible to extract the coincidence relations of the observed transitions. Extended results and their comparison to the largescale shell-model calculations will be presented in future publications.

This work was supported by the Research Council of Norway under project Grants Nos. 213442 and 240104 . V.W. was supported by the U.S. Department of Energy Grant No. DE-FG02-91ER-40609. 


\section{REFERENCES}

[1] T. Otsuka et al., Phys. Rev. Lett. 95, 232502 (2005).

[2] T. Otsuka, Phys. Scr. T152, 014007 (2013).

[3] K.T. Flanagan et al., Phys. Rev. Lett. 103, 142501 (2009).

[4] E. Sahin et al., Nucl. Phys. A 893, 1 (2012).

[5] S. Franchoo et al., Phys. Rev. Lett. 81, 3100 (1998).

[6] S. Franchoo et al., Phys. Rev. C 64, 054308 (2001).

[7] I. Stefanescu et al., Phys. Rev. Lett. 100, 112502 (2008).

[8] M. Doncel et al., Acta Phys. Pol. B 44, 505 (2013).

[9] E. Sahin et al., Phys. Rev. C 91, 034302 (2015).

[10] J.M. Daugas et al., Phys. Rev. C 81, 034304 (2010).

[11] C. Petrone et al., Acta Phys. Pol. B 44, 637 (2013).

[12] N. Patronis et al., Phys. Rev. C 80, 034307 (2009).

[13] S.V. Ilyushkin et al., Phys. Rev. C 80, 054304 (2009).

[14] T. Onishi et al., J. Phys. Soc. Jpn. 79, 073201 (2010).

[15] N. Fukuda et al., Nucl. Instrum. Methods Phys. Res. B 317, 323 (2013).

[16] T. Kubo, Nucl. Instrum. Methods Phys. Res. B 204, 97 (2003).

[17] T. Kubo et al., Prog. Theor. Exp. Phys. 2012, 3C003 (2012).

[18] S. Nishimura, Prog. Theor. Exp. Phys. 2012, 03 C006 (2012).

[19] P.-A. Söderström et al., Nucl. Instrum. Methods Phys. Res. B 317, 649 (2013). 